\title{
Lumbar Intraspinal Injection of Neural Stem Cells in Patients with Amyotrophic Lateral Sclerosis: Results of a Phase I Trial in 12 Patients
}

\author{
Jonathan D. Glass, ${ }^{a}$ Nicholas M. Boulis, ${ }^{\text {b }}$ Karl Johe, ${ }^{\text {c Seward B. Rutkove, }}$, Thais Federici, \\ Meraida Polak, ${ }^{a}$ Crystal Kelly, ${ }^{a}$ Eva L. Feldman ${ }^{\mathrm{e}}$ \\ ${ }^{a}$ Department of Neurology and ${ }^{b}$ Department of Neurosurgery, Emory University School of Medicine, Atlanta, \\ Georgia, USA; ${ }^{\mathrm{C}}$ Neuralstem, Inc., Rockville, Maryland, USA; ${ }^{\mathrm{d}}$ Department of Neurology, Beth Israel Deaconess

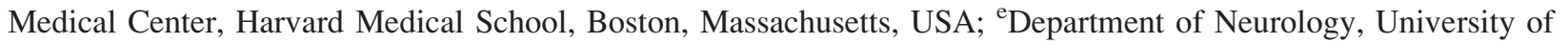 \\ Michigan, Ann Arbor, Michigan, USA
}

Key Words. Amyotrophic lateral sclerosis $\bullet$ Motor neuron disease $\bullet$ Stem cells $\bullet$ Spinal cord $\bullet$ Clinical trial

\begin{abstract}
Advances in stem cell biology have generated intense interest in the prospect of transplanting stem cells into the nervous system for the treatment of neurodegenerative diseases. Here, we report the results of an ongoing phase I trial of intraspinal injections of fetal-derived neural stems cells in patients with amyotrophic lateral sclerosis (ALS). This is a first-in-human clinical trial with the goal of assessing the safety and tolerability of the surgical procedure, the introduction of stem cells into the spinal cord, and the use of immunosuppressant drugs in this patient population. Twelve patients received either five unilateral or five bilateral (10 total) injections into the lumbar spinal cord at a dose of 100,000 cells per injection. All patients tolerated the treatment without any long-term complications related to either
\end{abstract}

the surgical procedure or the implantation of stem cells. Clinical assessments ranging from 6 to 18 months after transplantation demonstrated no evidence of acceleration of disease progression due to the intervention. One patient has shown improvement in his clinical status, although these data must be interpreted with caution since this trial was neither designed nor powered to measure treatment efficacy. These results allow us to report success in achieving the phase I goal of demonstrating safety of this therapeutic approach. Based on these positive results, we can now advance this trial by testing intraspinal injections into the cervical spinal cord, with the goal of protecting motor neuron pools affecting respiratory function, which may prolong life for patients with ALS. STEM Cells 2012;30:1144-1151

Disclosure of potential conflicts of interest is found at the end of this article.

\section{INTRODUCTION}

Disease-modifying options for patients with amyotrophic lateral sclerosis (ALS) are limited to treatment with riluzole, noninvasive positive pressure ventilation, and nutritional support, each having only a modest effect on disease progression and survival. Advances in stem cell biology have raised the question of whether cellular therapies may be a viable option for the treatment of ALS, either by replacing diseased cell populations directly or by introducing a cell population that can be supportive to the motor neurons affected by the disease process [1]. Several cell types have been proposed for testing in ALS, including mesenchymal stem cells (MSCs) derived from bone marrow, glial progenitor cells, and spinal cord-derived neural progenitor cells [2-4]. Each of these cell types has preclinical data both in vitro and in animal models supporting their potential as therapeutic options for ALS.
ALS patients have received implantations of MSCs into the spinal cord with reportedly few adverse outcomes [5], and a trial of intrathecal injections of MSCs for ALS is imminent (D. Offen, personal communication and http://www. brainstorm-cell.com).

Human spinal cord-derived stem cells (HSSCs) are neural progenitor cells isolated from the cervico-thoracic spinal cord of an 8-week gestation fetus [6]. These cells, expanded in serum-free media more than many passages, have shown therapeutic benefit when injected into the ventral horn area of spinal cord in the G93A rat model of familial ALS [7] and in a rat model of ischemic spinal cord injury [8]. In these motor neuron disease paradigms, the majority of the transplanted HSSCs differentiated into neuronal populations expressing glutamatergic and GABAergic neurotransmitter markers [9]. Very few of the transplanted cells were shown to have characteristics of alpha motor neurons. Even so, injection of HSSCs rescued endogenous motor neurons of G93A rats,

Author contributions: J.D.G.: site PI and primary writer of the manuscript; N.M.B.: surgeon; K.J.: sponsor of trial and developer of stem cell product; S.B.R.: responsible for EIM data; T.F.: protocol development and stem cell biologist; M.P.: lead clinical research nurse; C.K.: lead clinical research coordinator; E.L.F.: protocol development and overall PI.

Correspondence: Jonathan D. Glass, M.D., Department of Neurology, 101 Woodruff Circle, Suite 6000, Atlanta, Georgia 30322, USA. Telephone: 404-727-3507; Fax: 404-727-3157; e-mail: jglas03@emory.edu Received December 28, 2011; accepted for publication February 20, 2012; first published online in Stem Cells EXPRESS March 13, 2012. (C) AlphaMed Press 1066-5099/2012/\$30.00/0 doi: 10. 1002/stem.1079 
Table 1. Inclusion criteria

- Age 18 or older

- Sporadic or familial ALS diagnosed as laboratory supported probable, probable or definite according to the World Federation of Neurology El Escorial criteria [10]

- Capable of providing informed consent and complying with study procedures, including visit schedule

- Not pregnant or of child-bearing potential (females)

- Reside within driving distance to the study center with the ability to travel to the center for study visits

- Presence of a willing and able caregiver

- Medically able to undergo lumbar or cervical laminectomy ${ }^{a}$

- Medically able to tolerate immunosuppression regimen ${ }^{\mathrm{a}}$

- Not taking riluzole or on a stable dose for $\geq 30$ days

- Absence of chronic medical or psychiatric condition that may interfere with participation in the trial or add undue risk ${ }^{\mathrm{a}}$

Group A

- Inability to walk independently for $>2$ weeks due to weakness and/or spasticity; the ALSFRS-R lower extremity subscore $\leq 1$

- Vital capacity $\geq 60 \%$ of predicted measured in the seated position at the time of screening and $\geq 50 \%$ of predicted measured supine during the 7 days prior to surgery or stable for at least 3 months with tracheostomy and mechanical ventilation

Groups B and C

- Lower extremity weakness and/or spasticity, but able to walk independently. ALSFRS-R gait subscore $\geq 2$

- Vital capacity $\geq 60 \%$ of predicted measured in the seated position at the time of screening and $\geq 50 \%$ of predicted measured supine during the 7 days prior to surgery

${ }^{a}$ As determined by the site PI or the neurosurgeon.Abbreviations: ALS, amyotrophic lateral sclerosis; ALSFRS-R, ALS functional rating scale revised.

prolonged their survival, and induced partial motor recovery in rats with ischemic spinal cord injury [8]. These promising preclinical data led us to design a trial where spinal cord injection of HSSCs can be tested as a novel therapy for people with ALS.

In order to test any cell therapy for ALS, a safe and effective cell delivery system must be established, keeping in mind that people with ALS are already living with damaged nervous systems. Here, we report our experience with the injection of human spinal cord-derived stem cells (HSSCs) into the lumbar spinal cords of 12 ALS patients. This is a phase I study, focusing on the issues of safety surrounding this novel surgical technique and the implantation of HSSCs into people with ALS. In addition, we report on our experience with this type of trial design, including patient recruitment, presurgical and postsurgical monitoring, and identification of toxicities in this first-in-human clinical trial.

\section{Materials ANd Methods}

\section{Trial Design}

This is an ongoing phase I trial of spinal cord injections of neural stem cells. The protocol was designed to use the concept of "risk escalation," where the first patients would have the least function to lose should the spinal cord injections lead to serious complications of the transplant procedure. By design, each successive group of patients was exposed to increasing risk, both for the number of injections they received and the amount of neurological function they could potentially lose. Table 1 summarizes the clinical characteristics and surgical intervention for each group. The schedule required at least 1 month between each patient surgery.

\section{Neural Stem Cells}

The neural stem cells, NSI-566RSC, were isolated from the spinal cord of a single 8-week-old fetus, which for use in clinical trials were expanded, stored under current good manufacturing practice (cGMP) conditions, and supplied to the surgery site on the morning of surgery as a sterile suspension of live cells in a proprietary hibernation medium at $2^{\circ} \mathrm{C}-8^{\circ} \mathrm{C}$. All of the cell suspensions in the trial were prepared at
10,000 cells per microliter under a central cGMP facility and shipped by overnight delivery.

The characteristics of NSI-566RSC cells were previously described $[6,11,12]$. NSI-566RSC is a culture of human neural stem cells derived from a single source human fetal spinal cord tissue of approximately 8 gestational weeks and expanded serially by epigenetic means only. A region of the tissue corresponding to lower cervical/upper thoracic segments was dissected under a microscope. The associated meninges and dorsal root ganglia (DRG) were removed. From the clean single tissue, neural stem cells were isolated, pooled, and propagated as a single line. No genetic modification was applied to the cells at any point. Serial expansion was carried out as an adherent, monolayer culture in serum-free, animal-derived component-free, feeder cell-free conditions for approximately 60 population doublings in approximately 25 passages [11]. Human recombinant fibroblast growth factor (FGF-2) (basic fibroblast growth factor $[\mathrm{bFGF}]$ ) was used as the sole mitogen throughout. At various intermittent passages, the cells were harvested by trypsin treatment, which inactivates remaining bFGF and extracellular matrix proteins, and suitable cell aliquots were frozen and stored in liquid nitrogen until further use. A previously frozen lot of passage $4(\mathrm{P} 4)$ cells was qualified into a cGMP facility and used to generate the clinical lot used in this study. From approximately $1 \times 10^{7}$ frozen P4 cells, three-tiered cell banks were created under cGMP: a Master Cell Bank (MCB) at passage 6 consisting of $1.33 \times 10^{9}$ cells $(262$ vials at $5 \times$ $10^{6}$ cells per vial); a Working Cell Bank (WCB) at passage 9 consisting of $1.86 \times 10^{9}$ cells $\left(347\right.$ vials at $5 \times 10^{6}$ cells per vial); and a Clinical Cell Bank (CCB) at passage 12 consisting of $8.13 \times 10^{9}$ cells $\left(399\right.$ vials at $16 \times 10^{6}$ cells per vial). CCB was used for dosing as the clinical lot, without further culturing. One day prior to the scheduled surgery, one frozen vial of CCB was thawed, washed by centrifugation to remove the freezing medium, and concentrated to the desired target cell density of 10,000 cells per microliter in a hibernation medium free of preservatives and antibiotics. It was then shipped at $2^{\circ} \mathrm{C}-8^{\circ} \mathrm{C}$ to the surgery site by overnight delivery.

The MCB and WCB cells were $>99 \%$ nestin,$+ 0 \%$ glial fibrillary acidic protein positive (astrocyte marker), $0 \% \mathrm{NG} 2+$ (oligodendroglial marker), $0 \% \mathrm{TuJ} 1+$ (neuronal marker), $0 \%$ Iba1+ (microglial marker), 0\% smooth muscle actin+ (a marker of DRG-derived cells), $0 \%$ VE cadherin+ (a marker 
of vascular endothelial cells). The stability of the mitotic population was assessed by monitoring the population-doubling rate at each passage, which was reproducible at approximately 38 hours throughout the manufacturing process. Multipotent differentiation property of neural stem cells in MCB, WCB, and $\mathrm{CCB}$ was tested by in vitro differentiation for 6 8 days, which resulted in approximately $50 \% \mathrm{TuJ} 1+$ neurons and $50 \%$ glia for each bank, as previously reported as a hallmark of neuroepithelial stem cells [11]. The karyotypic stability was tested to be normal 46 chromosomes with XY at passage 4 and after further culturing of the CCB cells for two additional passages (passage 14). Tumorigenicity of the CCB cells was tested negative in colony-forming assays in soft agar, after subcutaneous implantation of $1 \times 10^{7}$ cells in athymic nude mice, and after 9 months of transplantation in spinal cord of naïve and contusion-injured nude rats (unpublished data). The WCB and/or CCB cells were tested negative for the following pathogens: bacteria, mycoplasma, in vitro adventitious viruses, bovine adventitious agents, porcine adventitious agents, retroviruses, human polyomavirus BK, human cytomegalovirus (CMV), Epstein-Barr virus, hepatitis A virus, hepatitis $\mathrm{B}$ virus, hepatitis $\mathrm{C}$ virus, herpesvirus type 6 , herpesvirus type 7 , herpesvirus type 8 , human immunodeficiency virus 1 and 2, human papillomavirus, T-cell lymphotropic virus, human polyomavirus JC, and human parvovirus B19.

NSI-566RSC was derived from a single donor and is intended as an allogeneic product. The cells in undifferentiated state and in differentiated state in vitro for up to 45 days express low levels of human leukocyte antigen (HLA)-A, HLA-DMB, and HLA-DRB1 mRNAs when probed by microarrays (Affymetrix U-133, data not shown). Other major histocompatability complex mRNAs were absent throughout the differentiation. By immunostaining with HLA-specific antibodies, no HLA class II antigens were detectible and less than $0.1 \%$ of the cells were positive for HLAA. The cells were negative for other HLA class I antigens. No immunoreactivity against $\mathrm{ABO}$ antigens was detectible.

NSI-566RSC was supplied as a suspension of live cells in a hibernation buffer free of preservatives and antibiotics, in an insulted shipping container that maintained the cell vials at $2^{\circ} \mathrm{C}-8^{\circ} \mathrm{C}$ for 7 days. Stability of the cells during shipping was tested and validated for period of 48 hours after the cell preparation to be usable for engraftment (data not shown). Before proceeding with surgery, the cells were inspected for viability using the method of trypan blue exclusion. The protocol called for at least $70 \%$ cell viability in order to proceed with the implantation; no other condition was required for administration into patients. In practice, viability ranged from $84 \%$ to $93 \%$, and there were no failed deliveries or rejections due to out-of-range release specifications. Cells that failed to exclude the trypan blue dye were considered "dead"; however, these cells could not be separated from the viable cells. After the final injection in each case, the cell suspension remaining in the syringe and tubing was dripped onto a glass slide and stained for morphological evaluation. In every case, this procedure assured us that the cells continued to be present within the suspension right through the final injection. Sterility and endotoxin level of each cell batch were verified by post hoc testing of retention sample kept at the manufacturing site, and the test result was notified to the study investigators within 14 days of surgery. Safety of this release procedure was validated at the cGMP facility by demonstrating no incidence of contamination in 10 successive runs. There was no incidence of nonconformance in regard to sterility or endotoxin level.

\section{Patient Selection}

The Emory University Institutional Review Board approved the trial design, patient eligibility criteria, and process of informed consent. Because this was a first-in-human trial for a disease with limited therapeutic options, we anticipated that a large number of patients would be interested in participation. The goals of the study and the inclusion criteria (Table 1) were clearly described and published on the Emory ALS Center website (als.emory.edu), and we instituted a web-based portal where patients could submit an "application" for admission to the trial. These submissions were reviewed on a weekly basis by the site principal investigator (PI) and study staff, and potentially eligible patients were invited for a screening visit at the study site. Patients and caretakers were asked to review the informed consent documents carefully before coming to the screening visit. During that visit, the site PI (J.D.G.) confirmed the diagnosis of ALS through standard clinical evaluation and repeat of any testing, if necessary.

Patients meeting inclusion criteria were engaged in a discussion of the risks of the trial with both the site PI (J.D.G.) and the neurosurgeon (N.M.B.). The informed consent document was reviewed with particular attention to the understanding that this phase I trial was designed only to test the safety of this new risky treatment, and there was neither expectation nor promise of therapeutic benefit. The discussions were videotaped to document the integrity of the informed consent process. In addition, a patient selection committee was inaugurated to oversee patient eligibility and to discuss the informed consent separately with the patient. The committee was composed of health care providers and community members who were not associated with the trial and was charged with confirming that each patient understood the goals of the trial, the potential severity of the risks, and that there was no expectation of clinical benefit.

\section{Presurgical Assessment}

Patients who met eligibility criteria and consented for participation were enrolled and assigned a unique patient identification number. These potential candidates were further screened with baseline laboratory studies to confirm that they did not have any condition that would exclude them from the study. Baseline clinical assessments included hand-held dynamometry (HHD), forced vital capacity (FVC), ALS functional rating scale-revised (ALSFRS-R) [13], and MRI scans of brain and entire spinal cord. HHD megascores were calculated from the individual measurements for each muscle [14], providing a normalized assessment of upper and lower extremity strength. In addition, a newer technique for assessing disease status, electrical impedance myography (EIM), [15] was performed bilaterally on gastrocnemius, tibialis anterior, and quadriceps, the muscles most likely to be impacted by the implantation. For purposes of identifying any major changes in progression postoperatively, all ambulatory patients (groups B and $\mathrm{C}$ ) were evaluated monthly for at least 3 months prior to surgery with HHD, FVC, ALSFRS-R, and EIM to generate a preoperative slope of disease progression that could be compared with postoperative measures [16]. Immunization against influenza, pneumonia, and herpes zoster was suggested for all patients but not required.

Each patient was re-evaluated clinically during the week prior to surgery to confirm that they still met inclusion criteria. These visits also included presurgical evaluation by the anesthetist as well as standard preoperative laboratories, electrocardiogram, and chest $\mathrm{x}$-ray.

\section{Surgical Procedure}

The surgical apparatus and injection procedures are described in previous publications [17-20]. Surgery was performed using standard anesthetic and monitoring techniques, including lower extremity somatosensory evoked potentials (all 


\begin{tabular}{lclr} 
Table 2. Patient groups & & & \\
\hline Group & $\begin{array}{c}\text { Patient } \\
\text { numbers }\end{array}$ & Characteristics & Treatment \\
\hline A1 & $1-3$ & Nonambulatory, FVC $>60 \%$ or trach/vent & 5 injections, unilateral \\
A2 & $4-6$ & Nonambulatory, FVC $>60 \%$ or trach/vent & 10 injections, bilateral \\
B & $7-9$ & Ambulatory, FVC $>60 \%$ & 5 injections, unilateral \\
C & $10-12$ & Ambulatory, FVC $>60 \%$ & 10 injections, bilateral \\
\hline
\end{tabular}

Abbreviation: FVC, forced vital capacity.

patients) and motor evoked potentials (patients 6-12). A twolevel, bilateral laminectomy was performed at vertebral level $\mathrm{T} 11$ and T12. The dura and arachnoid were opened and tacked to the surrounding soft tissues, and the surgical apparatus was attached to the vertebrae. A full description of the surgical apparatus and the injection procedure has been published [20].

Patients received either five unilateral or five bilateral (10 total) injections of HSSCs (Table 2). Each injection contained approximately 100,000 cells in an 8.5-10 $\mu$ l volume (concentration 10,000 cells per microliter). Cells were injected more than a 2-minute period, and the injection needle was kept in place for an additional minute to prevent reflux during needle exit. Total surgical time, from first incision to closing, ranged between 3 and 4.5 hours.

Postoperative care was standard for patients undergoing laminectomy with an intradural procedure. Patients were kept fully supine for at least 24 hours to prevent leak of cerebrospinal fluid. Pain was managed with i.v. and oral narcotics.

\section{Immunosuppression}

All patients received an i.v. dose of methylprednisolone (125 $\mathrm{mg}$ ) immediately prior to surgery and were treated postoperatively with oral prednisone $60 \mathrm{mg}$ tapering to 0 over 1 month. Two doses of basiliximab (20 mg i.v.) were given, one during surgery and another on postoperative day 4. Tacrolimus was given in b.i.d. oral dosing to maintain a trough level of 4 $8 \mathrm{ng} / \mathrm{ml}$, and mycophenolate mofetil was given at $1,000 \mathrm{mg}$ orally b.i.d. Tacrolimus and mycophenolate, if tolerated, will be given for the duration of the trial.

\section{Postoperative Assessments}

All patients were evaluated at 2 and 4 weeks postoperatively and then monthly thereafter. Standard clinical examinations and questionnaires were administered at each visit, along with laboratory measures of metabolic and hematologic function, serum levels for tacrolimus, and screening for reactivation of CMV by PCR in those patients with serological evidence of pre-existing infection. Each patient was also screened for the presence of HLA antibodies (panel reactive antibodies [PRAs]); a positive PRA screen was followed up with identification of specific antibody reactivity. Quarterly assessments included: ALSFRS-R, HHD, FVC, EIM, and bladder ultrasound for postvoid urine residual. MRI scans of the surgical region were performed every 6 months or more frequently if deemed necessary by the site PI.

\section{Study Oversight}

All clinical data were recorded both on paper and through electronic data capture. An experienced clinical trial auditor monitored all clinical data regularly at the study site, and the external safety monitoring committee reviewed all data on a monthly basis. Escalation of risk, that is, moving from one patient group to the next, required the approval of the safety monitoring committee.

\section{RESULTS}

\section{Recruitment}

We received requests for participation via direct interaction with patients at the Emory ALS Center, from phone calls, from letters and e-mails, and from our web portal. In all, 368 people, from 40 states and 18 countries, asked whether they could be part of the study. Even though the inclusion criteria were clearly outlined on the Emory ALS Center website, the majority of submissions were deemed ineligible because the patient did not live within driving distance of the trial site and/or the patient did not meet clinical criteria for participation. A total of 15 patients were invited to a screening visit, 12 who were ultimately enrolled. All patients who passed screening evaluation eventually underwent surgery-no patients became ineligible during the preoperative observation period.

The characteristics of the 12 patients are presented in Table 3. All patients were men, age range 37-66 years old and disease duration $1.5-13$ years. Three patients were chronically ventilated, two in group A1 and one in group A2. Selection of ventilated patients for these positions in the queue was intentional and was meant to reduce the risk of prolonged anesthesia should the surgeon require additional time during these initial surgeries, and to remove the risk of inability to extubate during these first-in-human surgical procedures. Since this trial was not designed to identify improvements in the course of ALS, the duration of disease was not considered as a criterion for participation. Indeed, four patients had a disease duration of $>5$ years at the time of surgery.

\section{Postoperative Course}

The details of the operative procedure, operative findings, and intraoperative electrophysiological monitoring will be presented in subsequent publications. To summarize, the operative time (first incision to last stitch) ranged from 3 to 4.5 hours. No major changes were identified in intraoperative evoked potentials. All nonventilated patients were extubated without problem in the operating room and had no immediate postoperative respiratory difficulties. Postoperative incisional pain was managed adequately with narcotic and non-narcotic analgesics. All patients returned home either $4(n=10)$ or 5 days after surgery. No patient required narcotics at the time of discharge.

\section{Adverse Events and Toxicities}

A summary of serious adverse events is presented in Table 4. Serious adverse events, defined as those requiring hospitalization or surgical intervention, were relatively few. In the opinion of the site PI and the study team, there were no adverse events or toxicities that could, with any certainty, be attributed to the HSSCs. Several patients experienced transient radicular-type pain and/or sensory abnormalities that were 


\begin{tabular}{|c|c|c|c|c|c|}
\hline Patient & Group & Gender & $\begin{array}{c}\text { Age at } \\
\text { surgery }\end{array}$ & $\begin{array}{c}\text { Disease duration } \\
\text { at surgery } \\
(\mathrm{yrs})\end{array}$ & $\begin{array}{c}\text { Implant } \\
\text { date }\end{array}$ \\
\hline 1 & A1 & Male & 61.7 & 5.2 (trach) & $1 / 20 / 2010$ \\
\hline 2 & A1 & Male & 43.4 & 12.7 (trach) & $3 / 12 / 2010$ \\
\hline 3 & A1 & Male & 51.1 & 2.1 & $4 / 14 / 2010$ \\
\hline 4 & A2 & Male & 37.5 & 2.0 (trach) & $5 / 26 / 2010$ \\
\hline 5 & A2 & Male & 66.2 & 2.2 & $6 / 23 / 2010$ \\
\hline 6 & A2 & Male & 55.0 & 2.2 & $8 / 18 / 2010$ \\
\hline 7 & B & Male & 59.0 & 1.6 & $10 / 20 / 2010$ \\
\hline 8 & B & Male & 41.1 & 5.5 & $11 / 17 / 2010$ \\
\hline 9 & B & Male & 54.5 & 3.5 & $12 / 29 / 2010$ \\
\hline 10 & $\mathrm{C}$ & Male & 48.9 & 11 & $2 / 9 / 2011$ \\
\hline 11 & $\mathrm{C}$ & Male & 39.4 & 1.5 & $3 / 9 / 2011$ \\
\hline 12 & $\mathrm{C}$ & Male & 65 & 3 & $4 / 13 / 2011$ \\
\hline
\end{tabular}

probably related to the injection procedure itself. One patient experienced severe bilateral groin pain following surgery, requiring narcotics. This pain resolved by the time of discharge from hospital. The radicular pains or sensory disturbances reported by the remaining patients were mild and transient.

One patient (number 4) developed a dural fistula and a spinal fluid leak, requiring reoperation and closure. This adverse event was definitely related to surgery and is believed due to the presence of an extradural drain placed at the time of surgical closure. This event led to a change in protocol and discontinuing the use of extradural drains. No further dural fistulas were encountered. Wound dehiscence requiring resuturing occurred in one patient (number 2).

MRI scans of the surgical region were done preoperatively and at 1, 6, and 12 months after surgery. The scans consistently showed accumulation of extradural fluid at the site of surgical invasion. These fluid collections showed no clinical correlate and resolved spontaneously. Representative examples of MRI images showing the extradural fluid collections are shown in Supporting Information Figure S1.

Other serious adverse events were: (a) a recurrent but transient encephalopathy that could not be attributed to surgery or cell implantation but may have been due to one or a combination of the immunosuppressant drugs (one occurrence); (b) hospitalizations for bronchitis and pneumonia that were likely due to the progression of ALS (two occurrences); and (c) two patients with pulmonary emboli occurring at 1 and 12 months postsurgery, respectively. The occurrences of pulmonary emboli were likely related to immobility and ALS [21].

There were two deaths. Patient 6 died suddenly and unexpectedly 8 months after surgery. He was doing well in terms of the clinical trial, although his ALS was certainly progressing. Autopsy revealed a congenital bicuspid aortic valve with ventricular hypertrophy. The cause of death was likely cardiac arrhythmia. Patient 3 died of progressive respiratory failure due to progression of his ALS 13 months following surgery.

The majority of nonserious adverse events were attributed to the toxicities of the immunosuppressant drugs. All patients tolerated the two doses of basiliximab and the month-long treatment with prednisone. However, only seven patients were able to tolerate the full doses of both mycophenolate mofetil and tacrolimus. Of the remaining five patients, three have stopped tacrolimus, two have stopped mycophenolate, and two have stopped both. Dose reductions to manage gastrointestinal (GI) toxicity (chronic diarrhea, abdominal bloating, and vomiting) were required in five patients. Two patients
Table 4. Serious adverse event

\begin{tabular}{lc}
\hline SAE name (related to study) & No. of subjects \\
\hline Transient encephalopathy & 1 \\
Pulmonary emboli & 2 \\
CSF leak & 1 \\
Wound dehiscence & 1 \\
Bronchitis/pneumonia & 2 \\
Dyspnea & 1 \\
Atrial fibrillation & 1 \\
Vomiting & 1 \\
Basal cell carcinoma & 1
\end{tabular}

Abbreviations: CSF, cerebrospinal fluid; SAE, serious adverse events.

remain on reduced doses of mycophenolate, one has returned to full dose, and two patients have stopped mycophenolate. The dose of tacrolimus was reduced in two patients due to vomiting and diarrhea, and three other patients have stopped taking tacrolimus. In all cases, it was difficult to decide which drug (mycophenolate or tacrolimus) was responsible for the GI symptoms. Dose reductions and stopping were done only after attempts to control GI symptoms with antigas and antidiarrheal agents.

\section{Markers of Cellular Rejection}

The HSSCs do not express HLA antigens; however, the HLA genotypes are known (Supporting Information Table S1). All patients were screened preoperatively for the presence of HLA antibodies (PRA test) and were retested at monthly intervals following surgery. At study entry, two patients had low prevalence class 1 HLA antibodies that were not matched to the HLA genotype of the donor stem cells. These participants continued to show these antibodies after surgery and did not generate any new antibodies with different HLA specificities. In the remaining 10 patients, including those who stopped immunosuppressive drugs, monthly testing for the presence of new HLA antibodies was consistently negative.

\section{Infection Due to Immunocompromise}

As part of the screening process, all patients were tested for evidence of present or past infection with hepatitis viruses, herpes 1 and 2, CMV and Epstein-Barr virus, HIV, and tuberculosis. Active infection was exclusionary (no occurrences). Evidence of previous CMV infection (seven patients) prompted the treatment with oral valganciclovir for 3 months following surgery. Monthly screens by PCR for the presence of CMV in blood have been negative in all seven patients. There were no clinically manifest occurrences of opportunistic infections.

\section{Outcome Measures}

Patients were followed monthly or bimonthly with standard clinical examination, FVC, ALSFRS-R, and HHD. These measures of disease status were collected at a single time point preoperatively for patients 1-6, and monthly for at least 3 months prior to surgery for patients 7-12. As shown in Figure 1, there was no obvious or consistent acceleration of disease progression for any patient as a result of the surgical procedure, introduction of HSSCs, or initiation of immunosuppressant treatment. Similarly, there was no consistent indication of a positive change in the slope of progression in patients 7-12, where preoperative clinical measures identified each patient's rate of decline. One possible exception is patient 11, whose measures of HHD and ALSFRS-R 

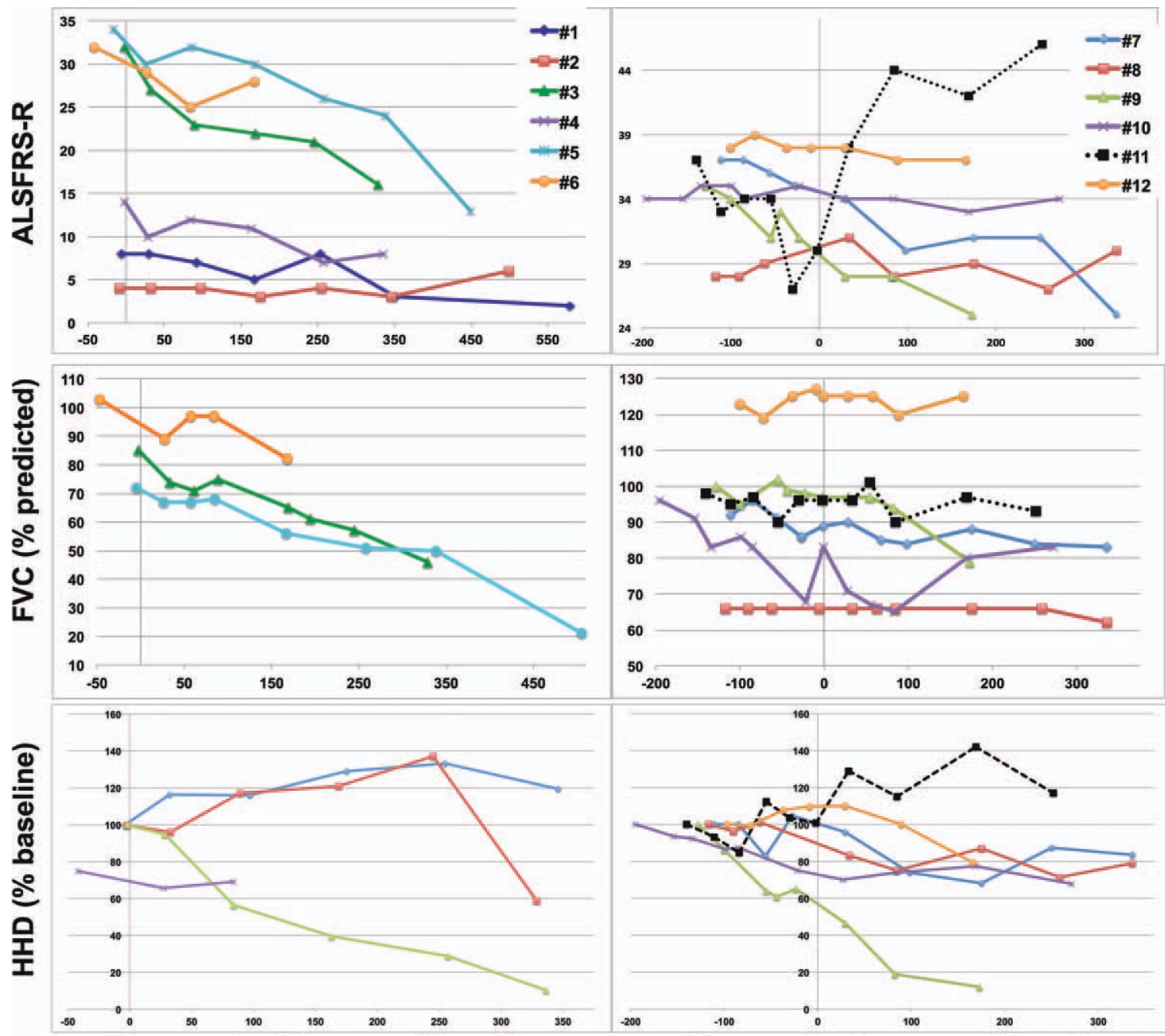

Days pre/post surgery (time 0 )

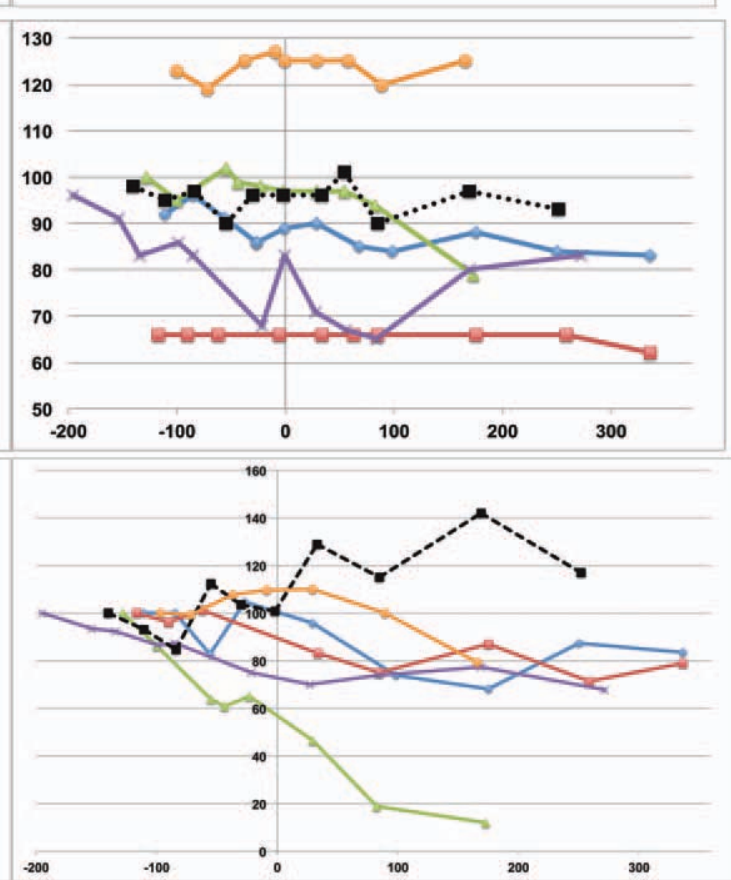

Days pre/post surgery (time $\mathbf{0})$

Figure 1. Quantitative evaluation of disease progression: left column patients 1-6, right column patients 7-12. Note that there was no precipitous decline in function after surgery for any patient. Patients 1,2, and 4 do not show FVC scores because they were on mechanical ventilation. Patients 1 and 5 did not have measurable HHD scores at any point. Note that patient 11 (dotted line) showed apparent improvement in ALSFRS$\mathrm{R}$ and HHD. HHD is shown as the composite "megascore" for lower extremities, normalized to the percent of the score at baseline. $x$-Axis is days presurgery or postsurgery (day of surgery $=$ day 0 ). Abbreviations: ALSFRS-R, amyotrophic lateral sclerosis functional rating scale-revised; FVC, forced vital capacity; HHD, hand-held dynamometry.

improved postoperatively. This finding must be interpreted with caution since there is no published experience with monthly clinical measurements for individuals with ALS. All published validations of these tools as measures of ALS progression are based on populations of patients, not on individuals, measured at $\geq 3$-monthly intervals. More frequent measurements of individual patients are likely to identify "noise" that may not reflect true changes in disease progression.

EIM, a newer and potentially more powerful biomarker of clinical progression, was performed at baseline for patients 16 and monthly for at least 3 months prior to surgery for patients 7-12. The data are summarized in Figure 2, in which the major parameter, the $50 \mathrm{kHz}$ phase averaged for all six muscles studied, for all 12 patients is plotted against time. These findings demonstrate the consistency of this measurement, and its ability to be used as a surrogate biomarker of disease progression, showing near linear changes in all those subjects who did not have end-stage disease and unchanging, very low values in those who did. No side-to-side differences in subsets of muscles were identified in those patients who had unilateral as compared to those who had bilateral injections.

\section{Discussion}

This phase I trial of spinal cord injections of neural stem cells was designed to test the safety and tolerability of this potential treatment in patients with motor disabilities due to ALS. We began with the most severely affected patients and progressed to patients with lesser disability, which we term as an "escalation of risk" trial design. Overall, we found that the surgical procedure was tolerated quite well, and that there were no identifiable toxicities attributable to the presence of the neural stem cells in the spinal cord. Thus, we consider this trial "successful" thus far, in terms of the stated design of testing safety in an ALS population.

Quantitative measures of disease progression were performed primarily to monitor whether patients, as a result of 


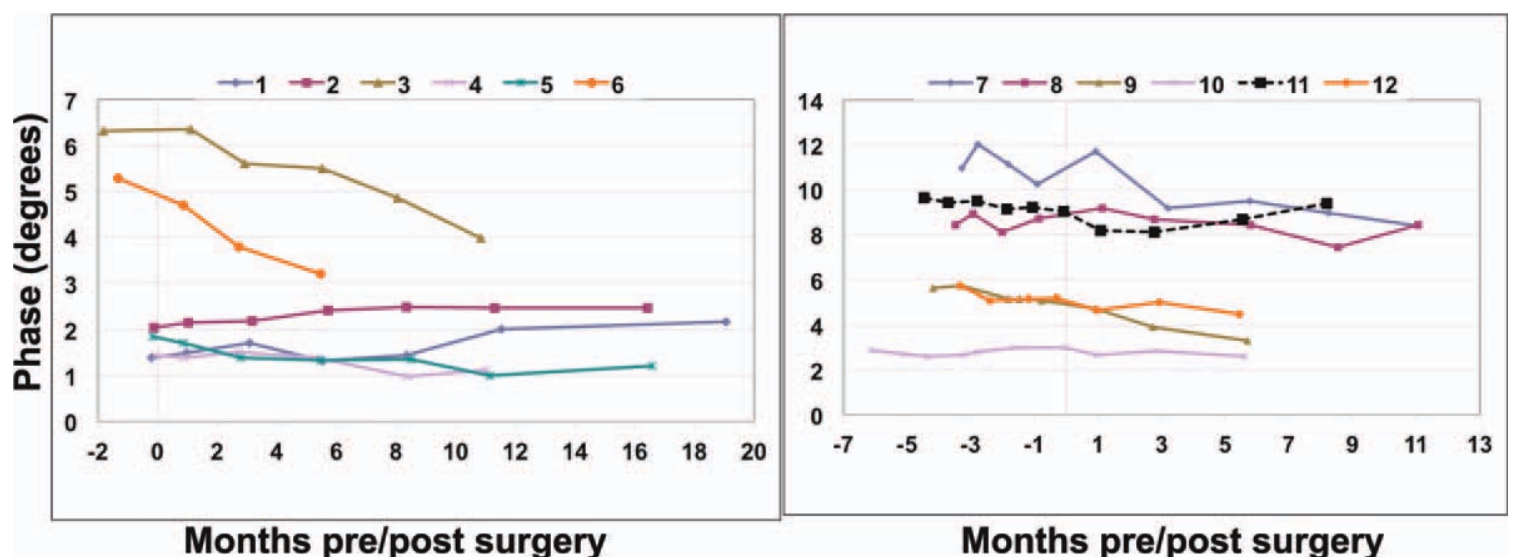

Figure 2. Disease progression as measured using electrical impedance myography (EIM). Average $50 \mathrm{kHz}$ phase for the six muscles studied (bilateral quadriceps, tibialis anterior, and medial gastrocnemius) in all 12 subjects. The lines represent linear fits of the data. Note again the consistent improvement in EIM score for patient 11 after surgery (dotted line).

the stem cell transplant, deteriorated from their preoperative course of disease progression. These data were more meaningful for patients 7 through 12, since this group was evaluated monthly for at least 3 months prior to surgery so the individual slopes of decline could be estimated. We did not perform these preoperative measurements for subjects 1 through 6 since these patients were nonambulatory and it was unreasonable to expect measurable changes in lower extremity strength, functional ability, or electrical impedance. As shown graphically in Figure 1 and confirmed by clinical assessment and the subjects' personal reports, there was no evidence for significant decline in function or acceleration of disease progression following surgical intervention and injection of the stem cells. An important finding was that our repeated measurements from HHD, ALSFRS-R, FVC, and EIM were quite consistent, suggesting that these assessments will be useful for future therapeutic trials of stem cell and other types of treatments.

There was, and continues to be, a major interest in whether the stem cell injections had any beneficial effect on the course of disease, even though the purpose of this phase I trial was to generate data on safety of the procedure. We cannot report with certainty that any patient either improved or plateaued in the rate of progression following surgery. However, the intensive and repeated monitoring of patients did show consistent positive changes in one patient. In patient 11 , the ALSFRS-R and HHD lower extremity megascore showed what appears to be significant improvement following surgery. Although exciting, these data demonstrating clinical improvement in one patient must be interpreted with caution. There is certainly no control group in this trial, and explanations other than an effect of the stem cells must be considered, including the possibility that the immunosuppressive regimen caused improvement. In addition, the repeated measurements of disease severity fluctuated, even prior to surgery, suggesting that any single measure of disease severity must be interpreted in the context of trends over several months. For example, patient 8 showed consistent improvement of ALSFRS-R for each of the 3 months leading up to surgery, which likely reflects variability of the measurement rather than true disease improvement. Each of the clinical measures, because they require subjective interpretation of function and/or physical effort on the part of the patient, is subjected to day to day variability. Indeed, the use of these measures for calculation of the rate of disease progression has only been validated for groups of patients evaluated at intervals of $\geq 3$ months, not for individual patients monitored monthly.

The quantitative assessment that showed the least "noise" between evaluations was EIM. In nonambulatory subjects 3 and 6, who did not have end-stage disease affecting the lower limb muscles, this measurement of muscle composition and structure showed steady decline after stem cell injection that was consistent with the other clinical measurements of disease progression. In patients with end-stage lower limb disease (patients 1,2, 4, and 5), consistent, relatively unchanged data were obtained during the entire study period. The EIM data in the ambulatory patients reflected the clinical decline in patients 7, 10, and 12. The remarkable improvement in ALSFRS-R and the lower extremity HHD megascore for patient 11 was also seen with EIM, although the improvement in EIM lagged behind the measures of ALSFRS-R and HHD. Since EIM is a measure of the integrity of the muscle itself and not the input of its parent motor neurons, changes in EIM are not expected to occur simultaneously with clinical performance of a muscle. Given the relative ease of application, the high intersession repeatability, and the lack of subjective effort required on the part the patient, the use of EIM as an outcome measure for future clinical trials should be considered, although EIM has not yet been validated as a marker of disease progression in a large longitudinal cohort of ALS patients.

The major toxicity for patients was related to the use of the immunosuppressant drugs tacrolimus and mycophenolate mofetil. The majority of patients either stopped one or both drugs, or required dose reduction due to GI symptoms of nausea, diarrhea, or anorexia. These toxicities are well known for both of these medications, limiting their use for a variety of clinical applications. One patient developed a skin cancer (basal cell carcinoma) that was cured with excision and may have been related to the immunosuppression. We did not encounter any major opportunistic infections associated with the immunosuppression, although three patients developed fungal skin infections in the groin region (tinea cruris).

The immunosuppression protocol was designed based on a standard protocol used for whole organ transplantation (i.e., kidney, heart, and liver). Since there was no previous experience with immunogenicity of transplantation of allogeneic fetal-derived stem cells into the nervous system, we decided to take a conservative approach at preventing rejection rather than risking the loss of cells due to immunogenicity. It is 
encouraging to note that the postoperative PRA screens at months 9-12 did not identify HLA antibodies to the HSSCs in any patient, even those who stopped immunosuppressant medications 1-3 months after transplantation because of GI toxicity. However, we will need to evaluate the survival of transplanted cells at autopsy, comparing patients on or off of immunosuppression at the time of death.

\section{Conclusions}

In summary, we have successfully treated 12 ALS patients with unilateral or bilateral intraspinal injections of neural stem cells. All patients tolerated the procedure without major surgical complications, and there are no indications to date that the stem cells themselves are either toxic or injurious to the spinal cord. Our quantitative clinical assessments showed no evidence of acceleration of disease following stem cell injections, meeting our stated goal of proving safety for this phase I trial. We have cautious optimism that a few of the patients may have slowed in their progression of lower extremity weakness, and one patient may have improved. Certainly, prolongation of life for patients with ALS will require therapeutic intervention at the level of the cervical spinal motor neurons affecting respiratory function. To reach this ultimate goal, we plan to move to injections into the cervical spinal cord, which is the next stage of this phase I trial.

\section{ACKNOWLEDGMenTS}

We thank the study participants and their families for their trust and their dedication to advancing ALS therapeutics. Thank you to Jane Bordeau and Latoya Shaw for help with patient assessments and to the staff of the Emory ALS Center. This study was funded by NeuralStem, Inc. Dr. Boulis is the inventor of devices to enable safe and accurate injection of the human spinal cord. Neuralstem, Inc. has purchased an exclusive license to this technology. Dr. Boulis received an inventors share of this fee and has the rights to royalty payments for distribution of this technology.

\section{Disclosure of Potential CONFLICTS OF INTEREST}

E.L.F. was supported by Novartis Pharmaceuticals (consultant/ advisory role) and J.D.G. has received research funding from Neuralstem, Inc.

\section{REFERENCES}

1 Lunn JS, Sakowski SA, Federici T et al. Stem cell technology for the study and treatment of motor neuron diseases. Regen Med 2011;6: 201-213.

2 Di Giorgio FP, Carrasco MA, Siao MC et al. Non-cell autonomous effect of glia on motor neurons in an embryonic stem cell-based ALS model. Nat Neurosci 2007;10:608-614.

3 Webber DJ, Minger SL.Therapeutic potential of stem cells in central nervous system regeneration. Curr Opin Investig Drugs 2004;5: 714-719.

4 Yu D, Silva GA.Stem cell sources and therapeutic approaches for central nervous system and neural retinal disorders. Neurosurg Focus 2008;24:E11.

5 Mazzini L, Vercelli A, Mareschi K et al. Mesenchymal stem cells for ALS patients. Amyotroph Lateral Scler 2009;10:123-124.

6 Guo X, Johe K, Molnar P et al. Characterization of a human fetal spinal cord stem cell line, NSI-566RSC, and its induction to functional motoneurons. J Tissue Eng Regen Med 2010;4:181-193.

7 Xu L, Yan J, Chen D et al. Human neural stem cell grafts ameliorate motor neuron disease in SOD-1 transgenic rats. Transplantation 2006; 82:865-875

8 Cizkova D, Kakinohana O, Kucharova $\mathrm{K}$ et al. Functional recovery in rats with ischemic paraplegia after spinal grafting of human spinal stem cells. Neuroscience 2007; 147:546-560.

9 Xu L, Ryugo DK, Pongstaporn T et al. Human neural stem cell grafts in the spinal cord of SOD1 transgenic rats: Differentiation and structural integration into the segmental motor circuitry. J Comp Neurol 2009;514:297-309.

10 Brooks BR, Miller RG, Swash M et al. El Escorial revisited: Revised criteria for the diagnosis of amyotrophic lateral sclerosis. Amyotroph Lateral Scler Other Motor Neuron Disord 2000;1:293-299.
11 Johe KK, Hazel TG, Muller T et al. Single factors direct the differentiation of stem cells from the fetal and adult central nervous system. Genes Dev 1996;10:3129-3140.

12 Yan J, Xu L, Welsh AM et al. Extensive neuronal differentiation of human neural stem cell grafts in adult rat spinal cord. PLoS Med 2007;4:e39.

13 Kollewe K, Mauss U, Krampfl K et al. ALSFRS-R score and its ratio: A useful predictor for ALS-progression. J Neurol Sci 2008;275:69-73.

14 Andres PL, Finison LJ, Conlon $\mathrm{T}$ et al. Use of composite scores (megascores) to measure deficit in amyotrophic lateral sclerosis. Neurology 1988;38:405-408.

15 Rutkove SB, Zhang H, Schoenfeld DA et al. Electrical impedance myography to assess outcome in amyotrophic lateral sclerosis clinical trials. Clin Neurophysiol 2007;118:2413-2418.

16 Leigh PN, Swash M, Iwasaki Y et al. Amyotrophic lateral sclerosis: A consensus viewpoint on designing and implementing a clinical trial Amyotroph Lateral Scler Other Motor Neuron Disord 2004;5:84-98.

17 Raore B, Federici T, Taub J et al. Cervical multilevel intraspinal stem cell therapy: Assessment of surgical risks in Gottingen minipigs. Spine (Phila Pa 1976) 2011;36:E164-E171.

18 Riley J, Butler J, Park J et al. Targeted spinal cord therapeutics delivery: Stabilized platform and MER guidance validation. Stereotac Funct Neurosurg 2007;86:67-74.

19 Riley J, Federici T, Park J et al. Cervical spinal cord therapeutics delivery: Preclinical safety validation of a stabilized microinjection platform. Neurosurgery 2009;65:754-761; discussion761-752.

20 Riley JP, Raore B, Taub JS et al. Platform and cannula design improvements for spinal cord therapeutics delivery. Neurosurgery 2011;69:147-155.

21 Gil J, Funalot B, Verschueren A et al. Causes of death amongst French patients with amyotrophic lateral sclerosis: A prospective study. Eur J Neurol 2008;15:1245-1251. 